\title{
Upregulation of Mad2 facilitates in vivo and in vitro osteosarcoma progression
}

\author{
LING YU ${ }^{1}$, SHIQING LIU ${ }^{1}$, WEICHUN GUO ${ }^{1}$, BO ZHANG ${ }^{1}$, YI LIANG ${ }^{2}$ and QISHENG FENG $^{2}$ \\ ${ }^{1}$ Department of Orthopedics, Renmin Hospital, Wuhan University, Wuhan, Hubei 430060; \\ ${ }^{2}$ State Key Laboratory of Oncology in Southern China and the Department of Experimental Research, \\ Sun Yat-Sen University Cancer Center, Guangzhou, Guangdong 510060, P.R. China
}

Received June 9, 2012; Accepted August 8, 2012

DOI: $10.3892 /$ or.2012.2032

\begin{abstract}
It has been reported that overexpression of $\mathrm{Mad} 2$ in transgenic mice leads to a wide variety of tumors, and Mad2 overexpression causes lung tumor relapse after oncogene withdrawal. In a previous study we demonstrated that Mad2 is abnormally upregulated in human osteosarcoma, however, the underlying mechanisms remain unknown. In this study, we found that transient Mad2 overexpression is sufficient to cause early dyscrasia and decreased survival in a xenotransplantation osteosarcoma mouse model, and Mad2 overexpression is associated with increased invasiveness and pulmonary metastasis. We also found that upregulation of Mad2 was accompanied by enhanced capability to self-renew. Our data validate the correlation between upregulation of $\mathrm{Mad} 2$ and osteosarcoma advancement, and that the underlying mechanisms involve the increase of invasiveness and cancer stem cell properties.
\end{abstract}

\section{Introduction}

Osteosarcoma is the most prevalent primary bone malignancy, and incidence peaks during the adolescent growth spurt (1). Numerous studies have sought to improve our understanding of osteosarcoma, and epidemiological studies have reported significant findings, such as the effects of puberty, disorders of bone growth and remodeling, and genetic predisposition (2-4). However, as with several types of cancer, the etiology of osteosarcoma remains unclear. Although neoadjuvant chemotherapy and limb salvage surgery greatly improve long-term

Correspondence to: Professor Shiqing Liu or Professor Weichun Guo, Department of Orthopedics, Renmin Hospital, Wuhan University, 238 Jiefang Road, Wuhan, Hubei 430060, P.R. China

E-mail: shiqingliu@whu.edu.cn

E-mail: guoweichun@yahoo.com.cn

Abbreviations: $\mathrm{Mad}$ 2, mitotic arrest defective protein 2; PI, propidium iodide; MTT, 3-(4,5-dimethylthiazol-2-yl)-2,5-diphenyltetrazolium bromide; FACS, fluorescence-activated cell sorting; FBS, fetal bovine serum; DMEM, Dulbecco's modified Eagle's medium

Key words: Mad2, osteosarcoma, invasiveness, metastasis, cancer stem cell survival and quality of life, recurrence and metastasis remain major challenges for clinicians.

Spindle assembly checkpoint provides a surveillance mechanism responsible for the fidelity of mitotic chromosome segregation $(5,6)$; it inhibits the onset of premature anaphase until all chromosomes are properly attached to the mitotic spindle apparatus and located at the metaphase plate, and defects in this process contribute to chromosomal instability and aneuploidy $(5,7,8)$.

Mad2 is a key component in the spindle assembly checkpoint (9). Our previous study demonstrated that Mad2 was overexpressed in human osteosarcoma tissue, and that increased expression of Mad2 was associated with early metastasis and poor survival (10). The findings of several reports are in accordance with our studies in many different malignant tumors $(8,11-13)$. The exact relationship between Mad2 expression and clinical outcome remains unclear.

In this study, we found that Mad2 overexpression conferred osteosarcoma cells with an enhanced ability to cause early dyscrasia, promote pulmonary metastasis and decrease survival. The underlying mechanisms involve an increased invasiveness and enhanced cancer stem cell-like properties.

\section{Materials and methods}

Chemicals and reagents. The following antibodies for immunoblot and immunohistochemistry analysis were purchased from Santa Cruz Biotechnology, Inc. (Santa Cruz, CA, USA): Nanog, POU5F1, Sox2, ABCG2 and $\beta$-actin. We obtained primary antibody of Mad2, CXCR4, MMP-1 from Abcam (Cambridge, MA, USA), and the horseradish peroxidase-labeled goat anti-rabbit IgG $(\mathrm{H}+\mathrm{L})$ were obtained from Cell Signaling Technology (Danvers, MA, USA). Anti-Stro-1 and CD117 primary antibodies used for FACScan analysis of cell surface expression were supplied by Becton-Dickinson Pharmingen (San Diego, CA, USA). B-27 serum-free supplement (50X) was obtained from Gibco (Grand Island, NY, USA). Recombinant EGF and bFGF were purchased from PeproTech (Rocky Hill, NJ, USA).

Cell culture. The human osteosarcoma cell line MNNG/HOS was purchased from the Shanghai Institute for Biological Sciences of the Chinese Academy of Sciences. All the cells were cultured in RPMI-1640 (Invitrogen, Carlsbad, CA, 
USA) supplemented with $10 \%$ (vol/vol) fetal bovine serum (FBS) (Invitrogen) and 1\% (vol/vol) penicillin-streptomycin (Invitrogen). Cells were propagated in a humidified environment at $37^{\circ} \mathrm{C}$ with $5 \% \mathrm{CO}_{2}$ and $100 \%$ humidity. Primary osteosarcoma cells were isolated from the xenotransplanted mice at the end of the observation point. In brief, tumor tissues were cut into small pieces and digested with $0.25 \%$ trypsin and $0.1 \%$ type II collagenase for $30 \mathrm{~min}$ and $2 \mathrm{~h}$, respectively. The released cells were cultured in RPMI-1640 medium supplemented with 10\% FBS and antibiotics. Cell viability was determined using trypan blue stain.

Adenoviral vector construction and infection. The AdV-Mad2-GFP was constructed by Shanghai GeneChem Co., Ltd. Titers of viral particles were determined according to Darling et al (14). For all experiments, cells were infected for $72 \mathrm{~h}$ at $37^{\circ} \mathrm{C}$ in $5 \% \mathrm{CO}_{2}$ using a multiplicity of infection (MOI) of 10 before subsequent detection. AdV-GFP infection was set as vehicle control, and a pseudo-infection acted as blank control.

Sarcosphere formation assay. The sphere formation assay followed procedures previously described (15). Fresh aliquots of EGF and bFGF were added every other day. After culturing for 14 days, colonies containing $>50$ cells were regarded as sarcosphere and the spheres were processed to form the next generation of spheres every 14 days for three more times.

Proliferation and drug toxicity assay. For proliferation detection, cells were seeded at $1 \times 10^{4}$ cells/well in 96-well plates for 2 , 4, 6 and 8 days and the cells were then harvested and counted. For drug resistance assay, cells were cultured $\left(5 \times 10^{4}\right.$ per well) in 96-well plates for 1 day, treated with increasing concentrations of doxorubicin and methotrexate for $24 \mathrm{~h}$ and then sent for MTT assay. MTT assay was performed according to the manufacturer's recommendations (Roche Diagnostics GmbH, Mannheim, Germany). Optical intensities were read on a multiwell scanning spectrophotometer at OD492 (Molecular Devices, Sunnyvale, CA, USA). All the experiments were repeated three times.

Transwell assay. Cell invasion assay was performed in dualchambered invasion plates (BD Biosciences, San Jose, CA, USA) as previously described. The polyethylene terephthalate membranes had an $8-\mu \mathrm{m}$ pore size and were coated with a layer of reconstituted extracellular matrix Matrigel. Assayed cells were placed in the upper chamber $\left(1 \times 10^{5}\right.$ cells/well $)$ in serum-free RPMI. The lower chambers were filled with RPMI medium with $10 \%$ FBS. At termination of the assay (24 h), the inserts were removed and the inner side was wiped with cotton swabs. The filters were stained with Harris's hematoxylin solution (Sigma-Aldrich, Inc., St. Louis, MO, USA) and were peeled off after washing and mounted on the slides. The migrated cells were counted under a light microscope.

Animals and orthotopic transplantation assay. To determine tumorigenicity and to establish orthotopic osteosarcoma animal models, 24 male BALB/C nude mice $~ 4-6$ weeks old were purchased from and maintained at the Wuhan University Center for Animal Experiment. The care and use of animals were in accordance with the recommendations and guidelines of the National Institutes of Health and were reviewed and approved by the Institutional Animal Care and Use Committee (IACUC) (approval number: 2011006). The mice were randomly divided into AdV-Mad2-GFP and AdV-GFP groups according to their injected cells, with 12 mice in each group. For orthotopic injections, cells (either transfected with AdV-Mad2-GFP or AdV-GFP) in log-phase growth were harvested, washed and resuspended with phosphate-buffered saline (PBS). BALB/C nude mice were anesthetized and cells $\left(5 \times 10^{5}\right.$ cells in $0.1 \mathrm{ml}$ PBS) were then injected into both distal femoral bone marrow cavities of each mouse. Mice were monitored daily until they reached the humane endpoint criteria. The criteria were defined as two months after injection or hunched abnormal posture for $>48 \mathrm{~h}$. Once the criteria were met, the mice were euthanized, and a section of the xenografted osteosarcoma tissue was obtained for primary cell culture; a second section was sent for pathological examination, and a third was sent for total-RNA and protein isolation for further molecular biological manipulation.

Western blots. For immunoblot analysis, cells were first infected with either empty AdV-GFP adenovirus or AdV-Mad2-GFP as described above; a pseudo-transfection group was set as blank control. Protein was extracted from subconfluent cultures using lysis buffer containing $1 \mathrm{mM}$ PMSF (Sigma-Aldrich, Inc.) and quantified using the BCA methods. Aliquots of $40 \mu \mathrm{g}$ protein from each sample were then resolved using SDS-PAGE gels and subsequently transferred to PVDF membranes. Membranes were blocked in $5 \%$ milk solution, incubated with primary antibody at $4^{\circ} \mathrm{C}$, overnight. They were then washed and incubated with horseradish peroxidase-conjugated secondary antibody. The immunoreactivity was detected by chemiluminescence. Statistical analyses of western blotting data were performed on the densitometric values obtained with NIH Image 1.61 software.

Immunohistochemistry. All tissues were subjected to routine pathological examination; in brief, the specimens were fixed and embedded. Serial sections $(5 \mu \mathrm{m})$ were cut and mounted on silane-coated glass slides. The sections were deparaffinized and rehydrated, then antigen retrieval was performed by boiling the slides in $10 \mathrm{mM}$ citrate buffer $(\mathrm{pH}$ 6.0) in a microwave oven for $10 \mathrm{~min}$. Endogenous peroxidase activity was blocked with $3 \%$ hydrogen peroxide for $30 \mathrm{~min}$. The slides were incubated in a humid chamber with respective primary antibodies, at $4^{\circ} \mathrm{C}$, overnight and were then incubated for $45 \mathrm{~min}$ at $37^{\circ} \mathrm{C}$ with horseradish peroxidase-conjugated secondary antibody. The slides were then developed using a DAB color kit (ProteinTech Group), counterstained with hematoxylin, and dehydrated. Primary antibodies were substituted with PBS as negative controls.

FACScan analysis of Stro-1 and CD117 expression. For analysis of cell surface markers, subconfluent cultures were infected as described above for $72 \mathrm{~h}$. The cells were harvested with fresh $0.25 \%$ trypsin solution (Sigma-Aldrich, Inc.) and then resuspended in PBS/0.5\% normal rabbit serum (Sigma-Aldrich, Inc.), and blocked on ice for $15 \mathrm{~min}$. Cells were subsequently labeled 

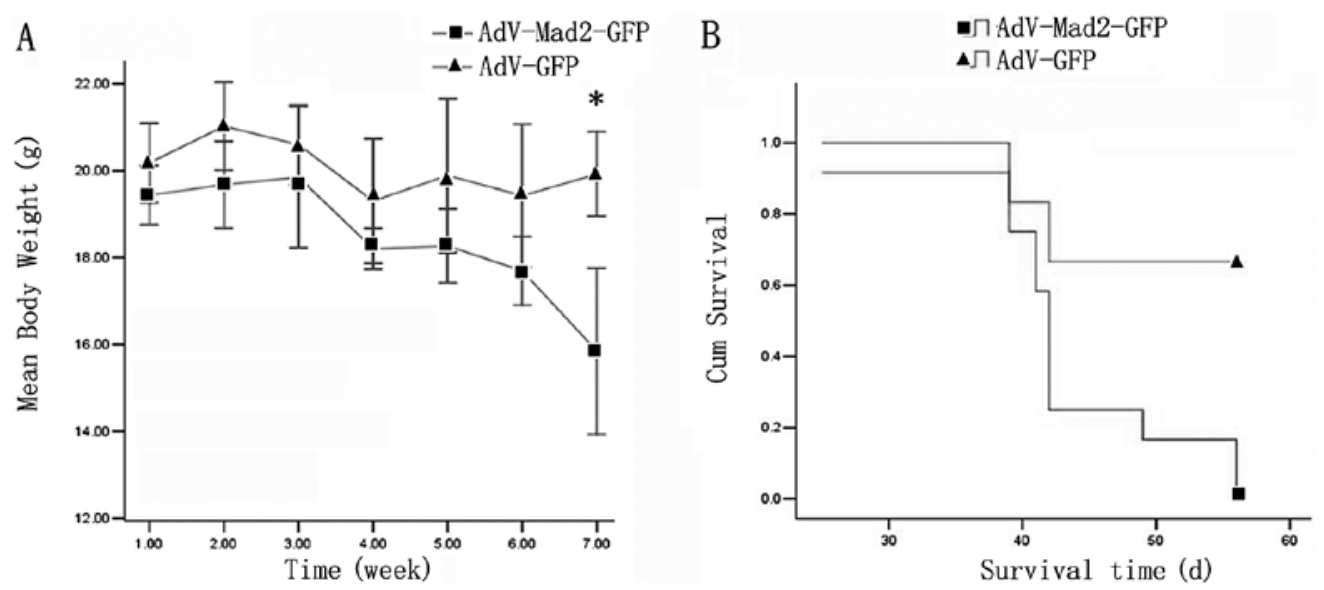

Figure 1. Early dyscrasia and decreased survival induced by Mad2 transient overexpression. (A) Curve for body weight of different groups at different times. The AdV-Mad2-GFP group showed early dyscrasia. (B) Survival curve for the two groups. The AdV-Mad2-GFP xenograft group showed poor survival.
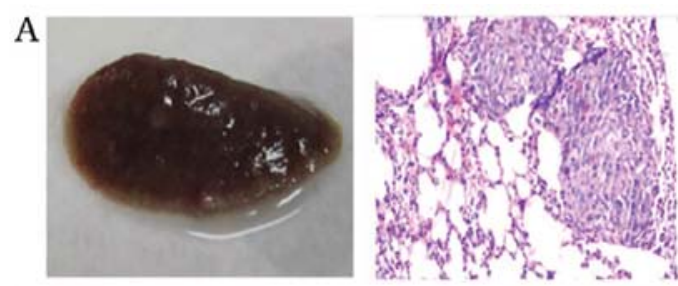

D Blank AdV-Mad2-GFP AdV-GFP

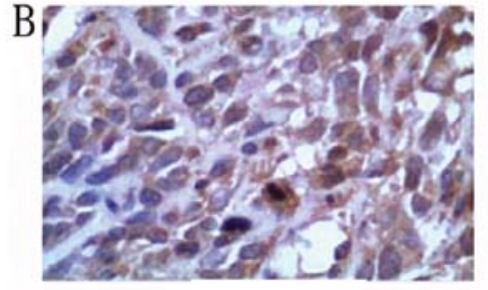

AdV-Mad2-GFP

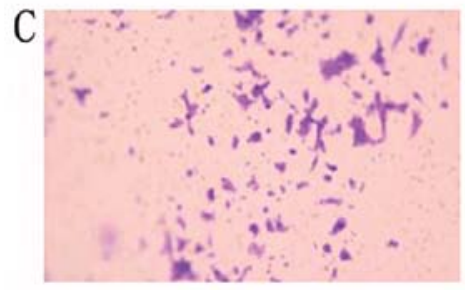

AdV-Mad2-GFP

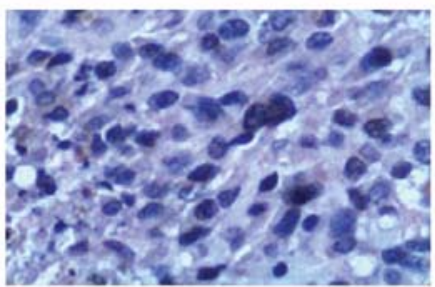

AdV-GFP

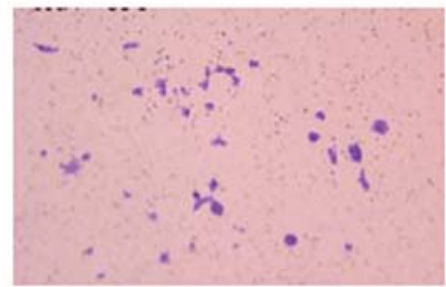

AdV-GFP

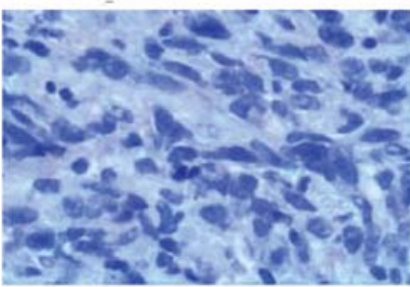

Negative control

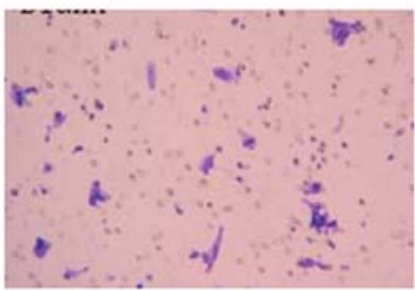

Blank

Figure 2. Mad2 overexpression promotes invasiveness and metastasis. (A) General appearance and H\&E staining of pulmonary micrometastasis in the AdV-Mad2-GFP xenograft group. (B) CXCR4 was upregulated in the primary tumor of the AdV-Mad2-GFP xenograft group by immunohistochemistry. (C) Transwell assay showed an increased ability to invade in the AdV-Mad2-GFP group following transient infection in vitro. (D) MMP-1 was upregulated in the AdV-Mad2-GFP group following transient infection in vitro.

with anti-Sto-1 and/or anti-CD117 for 60 min and maintained on ice until analysis. The expression was assessed by flow cytometry using a Becton-Dickinson FACSort (San Jose, CA, USA), and data were analyzed using WinMDI software (Scripps Research Institute, La Jolla, CA, USA).

Statistical analysis. Data are expressed as the mean \pm SD. Statistical analysis was performed by analysis of variance or Student's t-test using the SPSS13.0 statistical program with significance at $\mathrm{P}<0.05$. Survival was calculated by the KaplanMeier method, and the Log-rank test was performed with significance at $\mathrm{P}<0.05$.

\section{Results}

Mad2 upregulation promotes early dyscrasia and decreases survival. We found that the AdV-Mad2-GFP group exhibited early dyscrasia. The average body weight was $15.6 \pm 1.08 \mathrm{~g}$ for the AdV-Mad2-GFP xenografted group, while it was $\sim 20 \pm 2.52 \mathrm{~g}$ for the control group. We found that the AdV-Mad2-GFP xenografted group also exhibited early death; most of the mice from the AdV-Mad2-GFP xenograft group died at approximately the sixth week post-injection, whereas the mock group exhibited a higher survival rate at the terminal observation point (Fig. 1A and B). 
A
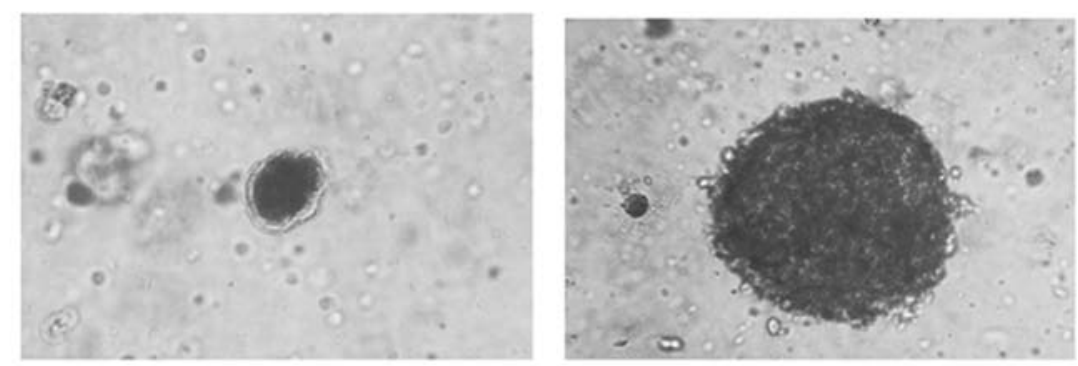

B

AdV-GFP
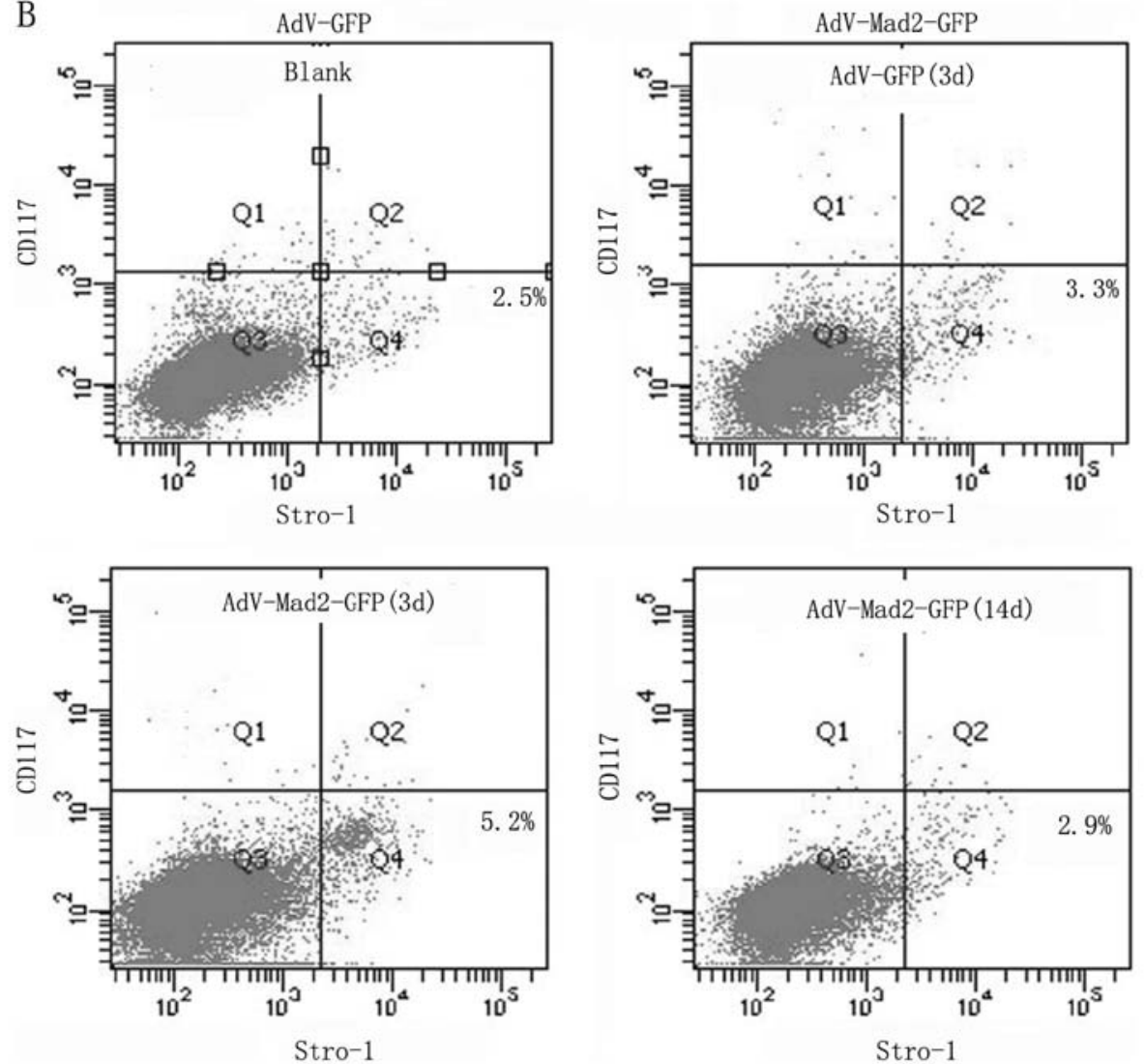

Figure 3. Enhanced stem cell properties after Mad2 overexpression. (A) AdV-Mad2-GFP infected cells were prone to form spheres. (B) Stro-1 positive cells were increased after transient infection of AdV-Mad2-GFP and the effect diminished by the 14th day after infection, however, there was no significant difference of CD117 positive cells among the different groups.

Transient Mad2 overexpression is associated with increased invasiveness and enhanced pulmonary metastasis. We observed an enhanced ability toward pulmonary metastasis in the AdV-Mad2-GFP xenograft group compared with the mock transfection group (Fig. 2A). In the mouse model, the CXCR4 expression was also upregulated in the primary tumor tissues from the AdV-Mad2-GFP xenograft group compared with the mock group (Fig. 2B). We further investigated whether Mad2 overexpression promoted invasiveness and found that AdV-Mad2-GFP cells exhibited an increased ability to invade following transient infection using the in vitro transwell assay (Fig. $2 \mathrm{C}$ ). Moreover, we found that MMP-1 was markedly upregulated in the AdV-Mad2-GFP group following transient infection (Fig. 2D).

Mad2 upregulation promotes stem cell-related properties. After 14 days of culture in sphere-specific conditions, the
AdV-Mad2-GFP group exhibited an enhanced ability to form spheres compared with the other two groups (Fig. 3A). The spherical cells were collected and subjected to the culture conditions above for three more generations, which confirmed the ability of these cells to self-renew. Stro-1 and CD117 were successfully used to identify osteosarcoma stem cells. Flow cytometry revealed that the AdV-Mad2-GFP group demonstrated an increased number of Stro-1 positive cells, whereas no effect was observed regarding CD117. The Stro-1-positive cells diminished 14 days after infection (Fig. 3B). In the present study, we did not find any significant change in the expression of stem cell-related genes among different infection groups using both qRT-PCR and western blot assay (data not shown).

Mad2 upregulation has no effect on osteosarcoma proliferation and drug resistance. We examined the proliferation 

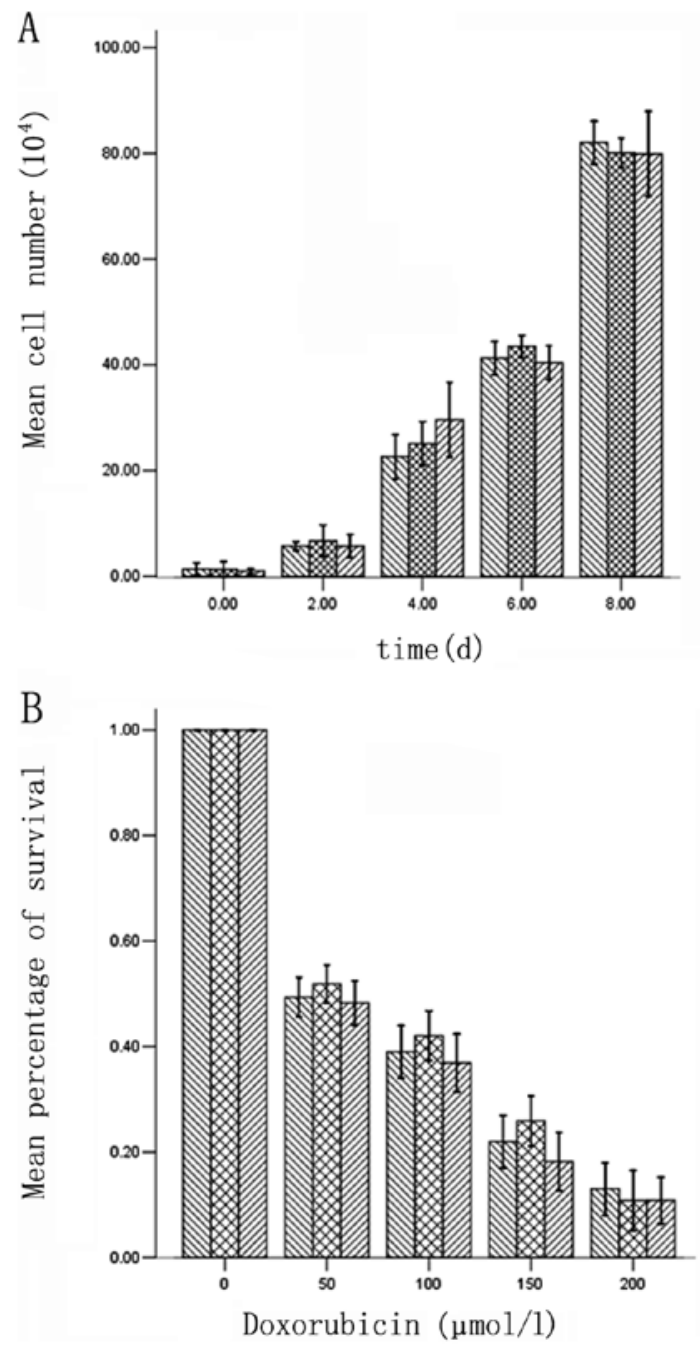

C
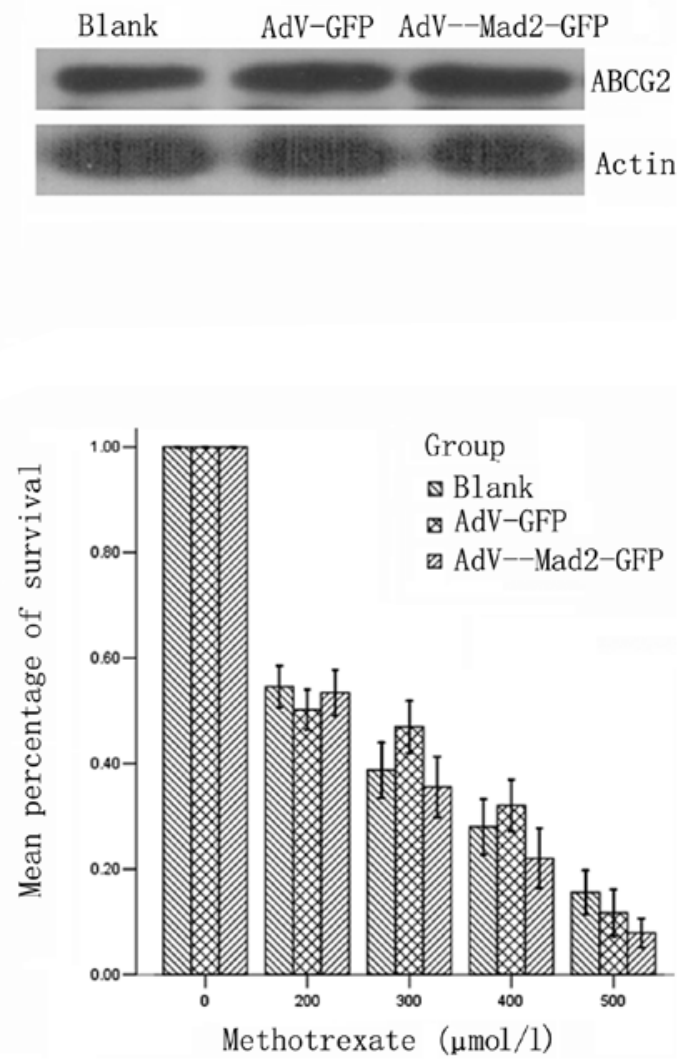

Figure 4. Mad2 overexpression had no effect on proliferation and drug resistance. (A) There was no difference in the proliferation curve among different in vivo transfection groups. (B) There was no significant difference of $\mathrm{IC}_{50}$ following transient infection. (C) Immunohistochemistry confirmed that the expression of ABCG2 was similar among different in vivo transfection groups.

Table I. Volume and weight of primary tumors in xenotransplantation models.

\begin{tabular}{llllll}
\hline & \multicolumn{2}{c}{ Volume $\left(\mathrm{cm}^{3}\right)$} & & \multicolumn{2}{c}{ Weight $(\mathrm{g})$} \\
\cline { 2 - 3 } \cline { 5 - 6 } Group & Mean $\pm \mathrm{SD}$ & P-value & & Mean $\pm \mathrm{SD}$ & P-value \\
\hline AdV-GFP & $1.792 \pm 0.273$ & 0.082 & & $2.628 \pm 0.450$ & 0.076 \\
AdV-Mad2-GFP & $1.656 \pm 0.262$ & & & $2.391 \pm 0.456$ & \\
\cline { 1 - 1 } & & & & &
\end{tabular}

SD, standard deviation.

potential among the different experimental groups, and found no significant differences (Fig. 4A). The similarity among the volumes and weights of the primary tumors in the different xenograft groups also confirmed our findings (Table I). We further investigated the drug-resistant properties and we did not find any significant differences following transient infection. The expression of ABCG2 also showed no significant differences (Fig. 4B and C).

\section{Discussion}

In order to upregulate Mad2 expression, we first examined the basal expression levels of Mad2 in osteosarcoma cell lines, and found that HOS/MNNG exhibited relatively low Mad2 expression and was tumorigenic, thus, it was suitable for our study (data not shown). We transfected the osteosarcoma cell line HOS/MNNG with AdV-Mad2-GFP and AdV-GFP, respectively. We used different concentrations of virus for infection, and found that transfection can be achieved with both low toxicity and high efficiency when using 10 MOI. The transfection efficacy was determined by fluorescence microscopy and flow cytometry three days post-infection, and the Mad2 expression was clearly elevated in the AdV-Mad2-GFP group compared with the other two groups (data not shown).

$\operatorname{Mad} 2$ is aberrantly expressed in many malignant types of cancer, such as gastric, colon, and lung cancer $(12,13,16)$. Our previous study demonstrated that $\mathrm{Mad} 2$ is overexpressed in human osteosarcoma and that increased expression of Mad2 correlated with a poorer clinical outcome. The transgenic animal model established by Sotillo et al was not tissue 
specific, therefore, they did not report any tumor primarily derived from bone (8). We utilized an orthotopic xenotransplanted mouse osteosarcoma model to detect whether Mad2 overexpression promotes osteosarcoma carcinogenesis. The mice were divided into two groups according to the different cells transplanted. In this study, we also found that Mad2 upregulation promoted early dyscrasia, enhanced pulmonary metastasis and decreased survival using a xenograft mouse model.

We found that Mad2 overexpression caused immediately enhanced invasive abilities, which we observed by the transwell invasion assay and MMP-1 expression levels following transient infection. We also found that, in the xenotransplantation model, CXCR4 was elevated in the tissue of the AdV-Mad2-GFP xenograft group compared with the control group. These results strongly support that $\operatorname{Mad} 2$ overexpression is involved in regulating invasion and metastasis.

Sotillo et al found that transgenic mice exhibiting Mad2 overexpression are more susceptible to tumor formation, accompanied by increased chromosome instability. Moreover, they reported that continued overexpression of Mad2 is not required for tumor maintenance, and overexpression of Mad2 in mice does not affect regression of Kras-driven lung tumors when Kras is inhibited; however, tumors that experienced transient Mad2 overexpression recurred at markedly elevated rates $(8,17)$. The above studies indicated that $\operatorname{Mad} 2$ is unlikely to act as an oncogene, thus, we further investigated the cancer stem cell hypothesis. Cancer stem cells possess the ability to self-renew, which is manifested by the serial formation of spheres in anchorage-independent serum free conditions. In our study, this property was promoted in the AdV-Mad2-GFP infection group.

Cancer stem cells share many cell surface markers with normal stem cells. Since Stro-1 is a traditional marker used to identify mesenchymal stem cells, it may be able to discriminate osteosarcoma stem cells from non-stem like cancer cells. Adhikari et al successfully isolated osteosarcoma stem cells that were Stro-1 and CD117 double positive (18). In our study, we found an increased percentage of Stro-1-positive cells in the AdV-Mad2-GFP infected group; however, we did not observe any significant changes in CD117 expression, for a reason that remains to be elucidated. We further detected the expression of many stem cell-related genes including Nanog, POU5F1 and Sox2; however, we did not find any significant changes (data not shown). We hypothesized that this is due to the relatively smaller portion of cancer stem cells, and western blot analysis is not an ideal technology under these conditions. Cancer stem cells possess the ability to differentiate. In the present study, we detected the population of Stro-1-positive cells 14 days after infection, and the percentage decreased to a level similar to the control group; since the adenovirus was not likely to recombine into the host genome, the effect gradually diminished, suggesting that Stro-1 positive cells again differentiated into Stro-1-negative cells when Mad2 decreased.

The role of Mad2 under normal conditions is to arrest mitosis in metaphase when abnormal chromosome aggregation occurs. We investigated whether Mad2 overexpression inhibits the cell cycle and therefore affects the potential for proliferation, and we found that there was no significant difference in the proliferation curve among the different groups.
The in vivo transplantation study demonstrated that primary tumor volume and/or weight exhibited no difference between the two groups; this finding also supports the possibility that Mad2 expression does not impact proliferation.

To investigate the drug-resistant properties further, cells were exposed to methotrexate and doxorubicin, two widelyused chemotherapy agents for osteosarcoma treatment. We found no significant difference after transient infection using MTT assay. ABCG2 is closely related to drug resistance, however, there was no evident difference between the different transfection groups.

Carcinogens not only induce gene mutations but also cause aneuploid lesions. Furthermore, normal cells exposed to chemical carcinogens can potentially become aneuploid $(19,20)$, and may become cancerous following the introduction of certain chromosomes $(21,22)$. However, only a fraction of these cells give rise to cancer, thus, it is possible that chromosomal derangements are important initial stages of tumor development, and may be involved in the advancement of cancer. The origin of cancer stem cells remains under investigation, and many researchers have demonstrated that the transformation of tissue by stem cells leads to cancer stem cell generation. In our study, we hypothesized that chromosome instability is a bridge between Mad2 overexpression and osteosarcoma stem cells, and this is in accordance with previous reports that indicated that genomic instability is another potential mechanism resulting in cancer stem cells (23).

In conclusion, our findings demonstrated that Mad2 overexpression exhibited a strong tendency toward metastasis and poor survival in a transplantation model. In the in vitro assay, we found that Mad2 upregulation closely correlated with invasiveness and stem cell-like properties, however, further investigation into the precise mechanism is warranted.

\section{Acknowledgements}

This study was supported in part by Grants from the Hubei Natural Science Foundation of China (302131702), and the Fundamental Research Funds for the Central Universities (no. 201130202020002).

\section{References}

1. Mirabello L, Troisi RJ and Savage SA: Osteosarcoma incidence and survival rates from 1973 to 2004: data from the Surveillance, Epidemiology, and End Results Program. Cancer 115: 1531-1543, 2009.

2. Savage SA and Mirabello L: Using epidemiology and genomics to understand osteosarcoma etiology. Sarcoma 2011: 548151, 2011.

3. Ottaviani G and Jaffe N: The etiology of osteosarcoma. Cancer Treat Res 152: 15-32, 2009.

4. Pritchard DJ, Finkel MP and Reilly CA Jr: The etiology of osteosarcoma. A review of current considerations. Clin Orthop Relat Res: 14-22, 1975.

5. Dobles $M$ and Sorger PK: Mitotic checkpoints, genetic instability, and cancer. Cold Spring Harb Symp Quant Biol 65: 361-368, 2000.

6. Silva P, Barbosa J, Nascimento AV, Faria J, Reis R and Bousbaa H: Monitoring the fidelity of mitotic chromosome segregation by the spindle assembly checkpoint. Cell Prolif 44: 391-400, 2011.

7. Weaver BA and Cleveland DW: Decoding the links between mitosis, cancer, and chemotherapy: The mitotic checkpoint, adaptation, and cell death. Cancer Cell 8: 7-12, 2005.

8. Sotillo R, Hernando E, Diaz-Rodriguez E, et al: Mad2 overexpression promotes aneuploidy and tumorigenesis in mice. Cancer Cell 11: 9-23, 2007. 
9. Eytan E, Braunstein I, Ganoth D, et al: Two different mitotic checkpoint inhibitors of the anaphase-promoting complex/ cyclosome antagonize the action of the activator Cdc20. Proc Natl Acad Sci USA 105: 9181-9185, 2008

10. Yu L, Guo WC, Zhao SH, Tang J and Chen JL: Mitotic arrest defective protein 2 expression abnormality and its clinicopathologic significance in human osteosarcoma. APMIS 118: 222-229, 2010.

11. Hisaoka M, Matsuyama A and Hashimoto H: Aberrant MAD2 expression in soft-tissue sarcoma. Pathol Int 58: 329-333, 2008.

12. Tanaka K, Nishioka J, Kato K, et al: Mitotic checkpoint protein hsMAD2 as a marker predicting liver metastasis of human gastric cancers. Jpn J Cancer Res 92: 952-958, 2001.

13. Wang L, Yin F, Du Y, et al: MAD2 as a key component of mitotic checkpoint: A probable prognostic factor for gastric cancer. Am J Clin Pathol 131: 793-801, 2009.

14. Darling AJ, Boose JA and Spaltro J: Virus assay methods: accuracy and validation. Biologicals 26: 105-110, 1998.

15. Gibbs CP, Kukekov VG, Reith JD, et al: Stem-like cells in bone sarcomas: implications for tumorigenesis. Neoplasia 7: 967-976, 2005.

16. Kato T, Daigo Y, Aragaki M, et al: Overexpression of MAD2 predicts clinical outcome in primary lung cancer patients. Lung Cancer 74: 124-131, 2011.
17. Sotillo R, Schvartzman JM, Socci ND and Benezra R: Mad2induced chromosome instability leads to lung tumour relapse after oncogene withdrawal. Nature 464: 436-440, 2010.

18. Adhikari AS, Agarwal N, Wood BM, et al: CD117 and Stro-1 identify osteosarcoma tumor-initiating cells associated with metastasis and drug resistance. Cancer Res 70: 4602-4612, 2010.

19. Duesberg P: Does aneuploidy or mutation start cancer? Science 307: 41, 2005.

20. Duesberg P, Fabarius A and Hehlmann R: Aneuploidy, the primary cause of the multilateral genomic instability of neoplastic and preneoplastic cells. IUBMB Life 56: 65-81, 2004.

21. Bachoo RM, Maher EA, Ligon KL, et al: Epidermal growth factor receptor and Ink4a/Arf: convergent mechanisms governing terminal differentiation and transformation along the neural stem cell to astrocyte axis. Cancer Cell 1: 269-277, 2002.

22. Ilyas M, Straub J, Tomlinson IP and Bodmer WF: Genetic pathways in colorectal and other cancers. Eur J Cancer 35: 335-351, 1999.

23. Liang Y, Zhong Z, Huang Y, et al: Stem-like cancer cells are inducible by increasing genomic instability in cancer cells. J Biol Chem 285: 4931-4940, 2010. 